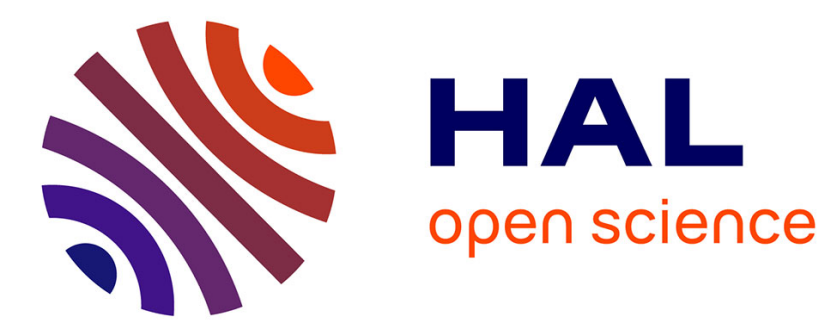

\title{
Experimental investigations on complex block propagation for the assessment of propagation models quality
}

Franck Bourrier, David Toe, Bruna Garcia, Julien Baroth, Stéphane Lambert

\section{- To cite this version:}

Franck Bourrier, David Toe, Bruna Garcia, Julien Baroth, Stéphane Lambert. Experimental investigations on complex block propagation for the assessment of propagation models quality. Landslides, 2021, 18 (2), pp.639-654. 10.1007/s10346-020-01469-5 . hal-03129220

\section{HAL Id: hal-03129220 \\ https://hal.science/hal-03129220}

Submitted on 2 Feb 2021

HAL is a multi-disciplinary open access archive for the deposit and dissemination of scientific research documents, whether they are published or not. The documents may come from teaching and research institutions in France or abroad, or from public or private research centers.
L'archive ouverte pluridisciplinaire HAL, est destinée au dépôt et à la diffusion de documents scientifiques de niveau recherche, publiés ou non, émanant des établissements d'enseignement et de recherche français ou étrangers, des laboratoires publics ou privés. 


\title{
Experimental investigations on complex block propagation for the assessment of propagation models quality
}

\author{
Franck Bourrier • David Toe • Bruna \\ Garcia · Julien Baroth · Stéphane \\ Lambert
}

Received: date / Accepted: date

\begin{abstract}
Rockfall propagation models are routinely used for the quantitative assessment of rockfall hazard. Their capacities and limitations remain difficult to assess due to the limited amount of exhaustive experimental data at the slope scale. This article presents experiments of block propagation performed in a quarry located in Authume (France). This study site was chosen for its complexity, related with the presence of topographical discontinuities and of configurations promoting block rolling. A total of more than one hundred blocks were released on two propagation paths. The propagation of the blocks was assessed by measuring the block stopping points as well as their kinematics at specific locations of the paths, called evaluation screens. Significant variability of the stopping points and of the block kinematics at the evaluation screens was observed and preferential transit and deposit zones were highlighted. The analysis of the results showed predominant effect of topography, in particular that related to topographical discontinuities. Significant influence of local and small scale parameters (e.g. block orientation, local topography) was also highlighted. These conclusions are of particular interest for researchers or practitioners who would like to assess the relevance of propagation modelling tools considering this complex study site. In this configuration,
\end{abstract}

Franck Bourrier

Univ. Grenoble Alpes, INRAE, ETNA, 38000 Grenoble, France

Univ. Grenoble Alpes, Inria, CNRS, Grenoble INP, LJK, 38000 Grenoble, France

E-mail: franck.bourrier@inrae.fr

David Toe

Univ. Grenoble Alpes, INRAE, LESSEM, 38000 Grenoble, France

Bruna Garcia

IMSRN, Ingenierie des Mouvements de Sol et Risques Naturels, 680 Rue Aristides Berges, 38330 Montbonnot, France

Julien Baroth

Univ. Grenoble Alpes, CNRS, Grenoble INP, 3SR, F-38000 Grenoble, France

Stéphane Lambert

Univ. Grenoble Alpes, INRAE, ETNA, 38000 Grenoble, France 
the quality of block propagation simulations should notably rely on the accuracy of digital terrain models, and on the integration of local conditions effects using physically based approaches.

Keywords Rockfall · Experiments · Block propagation models · Topography effects · Trajectory measurements

\section{Introduction}

The assessment of rockfall hazard, i.e. of rockfall triggering conditions and block propagation, is essential to develop mitigation strategies involving hazard zones determination and protection structures, in particular.

The analysis of rockfall triggering conditions requires field studies to identify prone-to-fall rock compartments and analysis of rockfall activity, given by historical inventories, surveys of deposited boulders or remote sensing methods, to assess triggering frequencies (Dussauge-Peisser et al. 2002; Guzzetti et al. 2003; Corominas et al. 2005; Rabatel et al. 2008; Agliardi et al. 2009; Hantz 2011; Abellan et al. 2010; Dewez et al. 2013).

Block propagation can be evaluated at the district scale based on empirical approaches that estimate the maximum block propagation distance using historical surveys of rockfall events. Among these approaches, the commonly used energy line method, initially developed by Heim (1932), assesses the maximum propagation distance from the intersection between the topography and a line passing by the top of the cliff, characterized by its angle with the horizontal direction (Jaboyedoff and Labiouse 2011). Complementary, block propagation models are routinely used for the quantitative assessment of rockfall hazard (e.g. Dincer et al. (2016); Sarro et al. (2018); Macciotta and Martin (2019)) and their use is almost exclusively limited to studies on small areas. In these models, one of the major difficulties is the development of physically consistent and field applicable approaches to model the interaction between the block and the natural terrain. Two types of propagation models exist. They either consider the block as a single material point or explicitly account for the fragment shape (Bourrier and Hungr 2013; Bourrier et al. 2012). Although both types of approaches provided representative results on different study sites (Jaboyedoff and Labiouse 2011; Labiouse and Heidenreich 2009; Guzzetti et al. 2003; Lu et al. 2019), several limitations of these models have been identified in the literature. The more commonly reported ones are limited capacities to model block propagation for complex topographies, e.g. ledges due to road building or complex 3D topographies (Bourrier et al. 2009; Lambert et al. 2013), and for block motion almost analogous to rolling, e.g. propagation on gentle slopes or soft soils (Bourrier and Hungr 2013; Spadari et al. 2012).

Various experiments have been conducted in view of analyzing the interactions between the block and the slope surface, the block propagation processes at the slope scale, as well as for calibrating and evaluating the predictive capacities of propagation models. These studies were conducted either in the field (Giani et al. 2004; Dorren et al. 2006; Bourrier et al. 2009, 2012; Spadari 
et al. 2012; Caviezel et al. 2019) or in laboratory conditions (e.g. Chau et al. (1998); Labiouse and Heidenreich (2009); Hu et al. (2017); Asteriou and Tsiambaos (2018); Garcia et al. (2017)). Most of these experiments focused on the detailed analysis of few block rebounds or block trajectories, or on the effect of specific parameters (e.g. block shape) for specific propagation conditions (e.g. propagation as a succession of flight and rebound phases). In addition, only few of these studies provide substantial and exhaustive data on blocks propagation at the slope scale. In particular, data concerning both block stopping positions and kinematics are scarce, specifically for study sites where block propagation is complex to model because of problematical topography or propagation processes.

In practice, the limited amount of available exhaustive and accurate experimental data at the slope scale entails that most of propagation models are not calibrated exhaustively and thoroughly. For a particular study site, the assessment of the accuracy of propagation simulations is limited to the analysis of the simulated stopping points of the blocks in comparison with past-events, mainly because the kinematics of the blocks cannot be easily quantified from the analysis of past events. Consequently, the assessment of the relevance of block propagation simulation works is challenging. These simulation works strongly depend on the expertise of the operator, because of incomplete model calibrations, based on scarce data available, generally limited to few past events in the study site.

In this context, the objective of this study was to provide and analyze experimental data of block propagation for the assessment of propagation models relevance on a configuration where propagation simulations is potentially problematical. For that purpose, an experimental campaign involving exhaustive measurements of block propagation on a complex study site has been designed to provide exhaustive and statistically representative data for assessing the model capacities and identifying their potential limitations for complex study sites.

First, the characteristics and the complexity sources of the study site as well as the experimental protocol are presented. Second, the experimental results are exposed in detail, considering the various data recorded. Finally, these results are discussed focusing on the analysis of the prevailing effects leading block propagation and highlighting the difficulties in modeling these trajectories.

\section{Experiments}

The study site was chosen because its topography and slope surface materials were prone to induce complex block propagation processes. Specific attention was also paid to set up controlled experimental conditions in order to quantify the parameters classically identified in the literature for having an effect on block propagation (release conditions, block mass and shape, local variability of soil properties, in particular). Finally, one major concern was the acquisition 


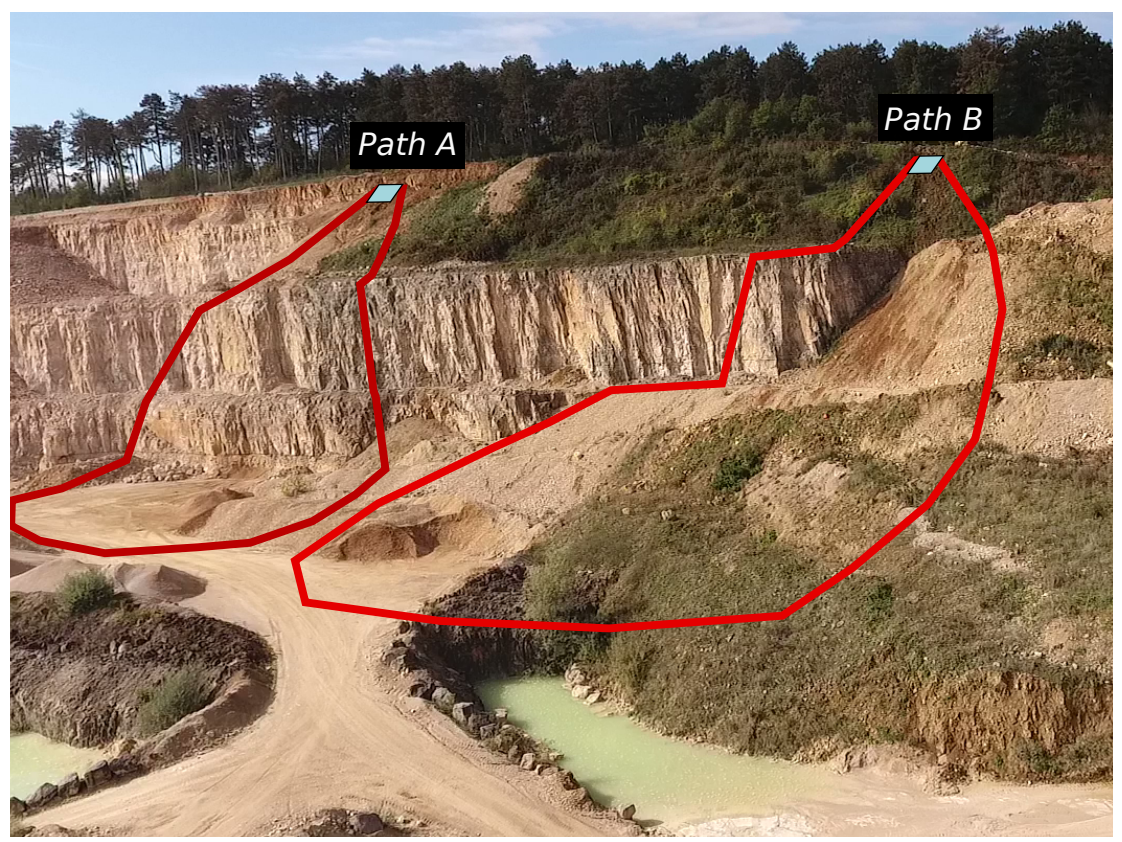

Fig. 1 Overview of the study site. The release zones correspond to the blue polygons and the potential block propagation zones to the red contours.

of a significant amount of data with sufficient accuracy despite non negligible constraints of safety and practical feasibility. The details of the experiments are presented in the following.

\subsection{Study site}

The study site, located in a quarry (Authume - France, owner: Pernot S.A, Figure 1), was selected because it offers significant complexity and variability in terms of topography and surface characteristics. The propagation paths were chosen to favor challenging-to-model block trajectories. The two selected paths exhibit combinations of gentle slopes and soft soils, favoring block motion almost analogous to rolling, and complex topographies with rapid changes in slope inclination and/or orientation. These paths are described in the following. The soils are characterised considering classifications commonly used in trajectory simulation codes that are available to design engineers.

The first profile, referred to as Path $A$ (Figures 1 and 2), is characterized first by a gentle slope section, $30 \mathrm{~m}$ in length along the horizontal axis with a $32^{\circ}$ inclination. The slope material is made of dry newly deposited quarry waste, mixing sand, clay and limestone fragments. This upper slope is terminated by a flatter and rougher part where boulders are more numerous, overhanging a $18 \mathrm{~m}$ in height subvertical rock cut inclined by $10^{\circ}$ towards the vertical and 

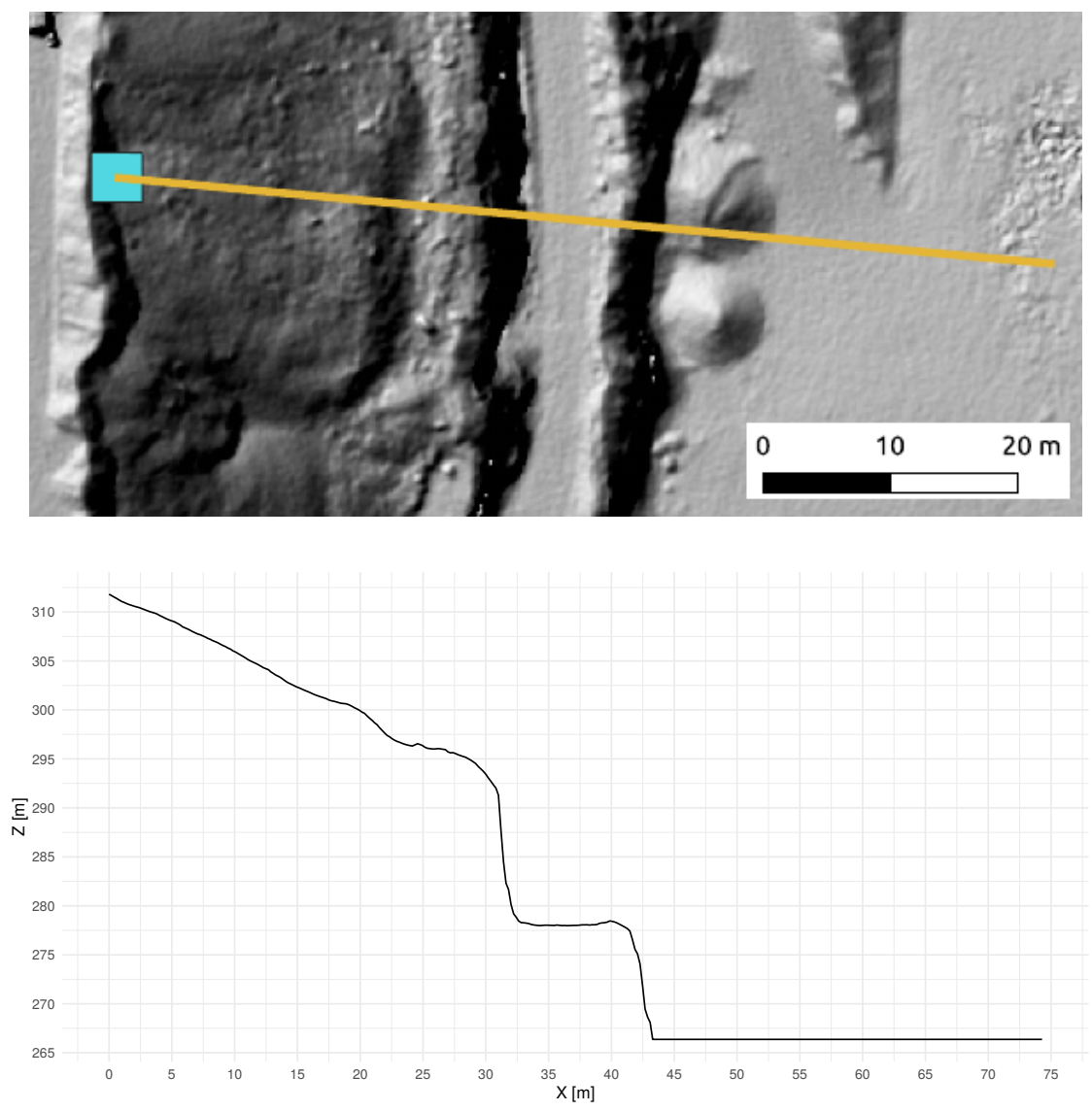

Fig. 2 2D profile along the main rockfall path, for Path $A$.

made of compact limestone rock. The $7 m$ in width horizontal track at the toe of this wall consists of compact quarry waste. A second $13 \mathrm{~m}$ in height rock cut, with same characteristics as the first one, separates this track from the quasi-horizontal platform acting as terminal deposit area. The slope profile along the perpendicular to the steepest slope direction is rather uniform.

On such a path, numerous block trajectories are possible. A block could directly stop on the uphill gentle slope just after release or on its terminal flatter part. It could also propagate on the gentle slope, fall on the intermediate track, and stop or propagate down to the platform. Alternatively, when reaching the upper ledge on top the first rock cut, it could directly bounce over the intermediate track and directly reach the platform. None of these potential trajectory archetypes can be a priori identified as preferential mainly because the propagation on the uphill slope is difficult to predict by expert knowledge only because its low inclination and soil mechanical characteristics limit block rebound. 

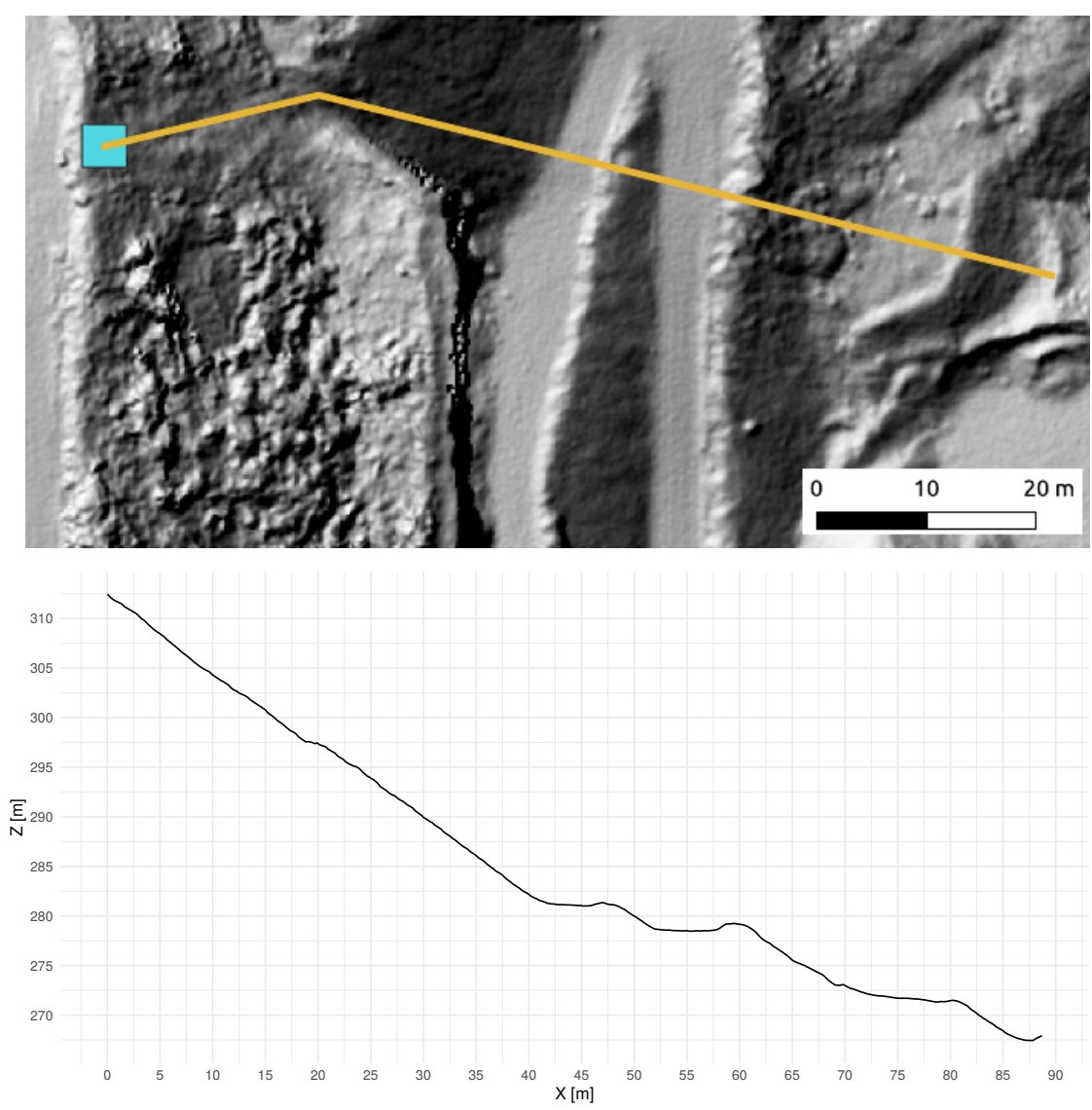

Fig. 3 2D profile along the main rockfall path, for Path $B$.

The second profile, referred to as Path $B$ (Figures 1 and 3 ) is characterized in its upper section by a $32^{\circ}$ in inclination slope, $40 \mathrm{~m}$ in length along the horizontal axis. At mid-distance, the path is bordered by a rock cut on one side and by a talus on the other. This creates a so-called corridor. The soil in this upper section is made of medium soft quarry waste, mixing sand, clay and limestone fragments. Above the corridor, the soil is revegated while it was newly deposited within the corridor.

The intermediate section of $P$ ath $B$ is characterised by two successive tracks $7 m$ in width each. These tracks exhibit slight inclinations in opposite directions and are separated by a slope inclined by $32^{\circ}$ which is made of medium soft quarry waste. The second track is followed by a $27 \mathrm{~m}$ in horizontal length and $32^{\circ}$ in inclination slope also made of medium soft quarry waste, terminated by a globally horizontal deposit area.

This path was chosen because of potentially substantial 3D effects in the blocks trajectories, as compared to Path A. Such effects were expected to 
result from the corridor in the second half of the upper section, creating a preferential propagation path, and from the presence of the slightly sloping tracks perpendicular to the mean steepest direction in its intermediate section, favouring lateral deviation.

For both paths, the tracks are bordered by a rock pile forming a more or less rounded berm, typically $0.5 \mathrm{~m}$ in height.

These two paths thus exhibit significantly different topographical features. Path $A$ is characterized by two subvertical rock cuts, compared to Path $B$ mainly showing slight changes in slope inclination. Along the perpendicular to the steepest slope direction, Path $A$ is rather uniform contrary to Path $B$. These differences are expected to result in different blocks kinematics evolution along these two paths. For example, high amplitude topographical discontinuities favor the occurrence of normal impacts and are thus expected to induce higher block kinetic energy dissipation as well as complex rebounds involving couplings between the components of the velocity (Bourrier et al. 2012).

Overall, the two paths complement one another. Path $A$ focuses on the effects of topographical discontinuities, gentle slopes, and soft soils, and Path $B$ allows to investigate the influence of complex 3D topography in addition to the previous effects.

\subsection{Experimental and measurements protocols}

\subsubsection{Experimental procedure}

Preliminary experiments, consisting of ten block releases on each propagation path, were first conducted to identify optimal block release conditions, to set up the experimental and measurement protocols, and to design the data analysis procedure.

The main experiments consisted in the successive release of approximately fifty blocks on each propagation path using a power shovel. The number of blocks released on each profile corresponds to the larger amount of releases possible given the limited duration of the experiments. However, it is not sufficient to guarantee statistical representativeness for all the quantities explored, especially for the extreme quantiles of quantities distributions.

A release zone ( $4 m \times 4 m$ horizontal area) was delimited at the top of each path. The vertical release heights were set at $4 m$ for Path $A$ and $2 m$ for Path $B$. The height was larger for Path $A$ to prevent block stopping directly on the uphill gentle slope, as observed in the preliminary experiments.

\subsubsection{Block characteristics assessment}

The blocks were extracted from the quarry and visually selected to obtain block volumes approximately ranging between 0.1 and $0.75 \mathrm{~m}^{3}$. The weight of each block was measured using a piezoresistive force sensor (Chatillon SLC10000 accuracy $\pm 20 \mathrm{~kg}$ ). Each block was installed in a wire mesh basket connected to 

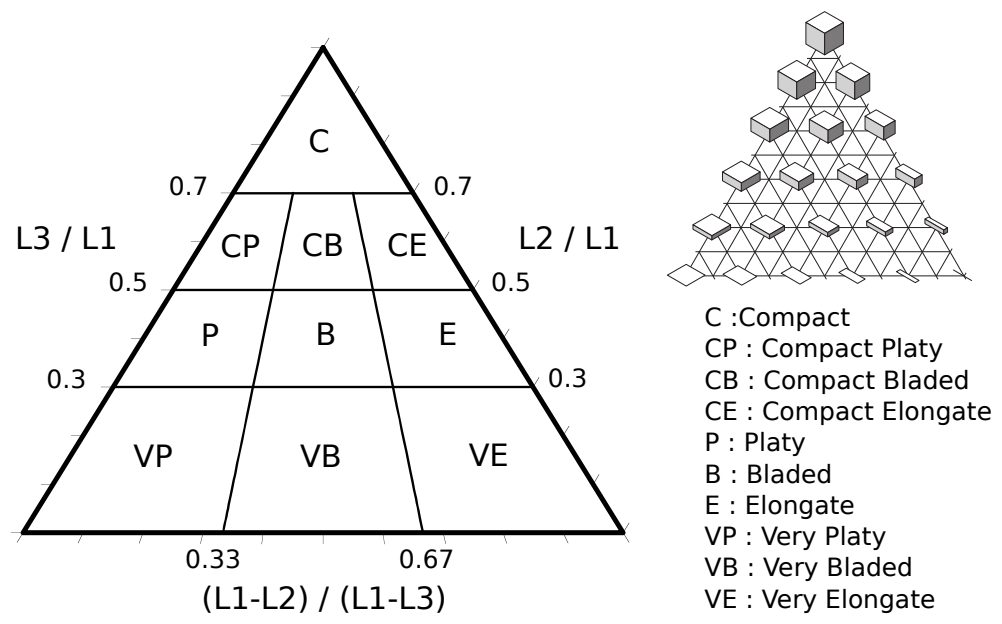

Fig. 4 Shape classification diagram (adapted from Blott and Pye (2008)).

the force sensor. The block was weighed lifting up the sensor using the crane of a power shovel.

Additionally, three principal lengths have been measured for each block. These lengths characterize the minimal parallelepiped of dimensions $L_{1}, L_{2}$ and $L_{3}$, with $L_{1}>L_{2}>L_{3}$ that incorporates the block. This data was used for block shape characterisation according to the classification proposed in Sneed and Folk (1958), which was recently adapted for the assessment of block shape in the field of rockfall (van Veen et al. 2017; Bonneau et al. 2019). In this classification (Figure 4 - adapted from Blott and Pye (2008)), four main types of block shapes are differentiated : compact, platy, blade and elongate. Blade and elongate shapes potentially favour high rebounds compared to the others (compact and platy) (Labiouse and Heidenreich 2009; Glover 2015) while platy blocks have been identified in the literature (Glover 2015; Leine et al. 2014) for their predisposition to travel further by rolling motion.

Data concerning the blocks mass and shape are given in the section dedicated to the influence of these parameters on the block propagation.

\subsubsection{Block trajectories assessment}

Precise determination of the blocks stopping point location was conducted after each series of five blocks release to limit the duration of the experimental campaign. The theodolite used for this purpose (Leica TS02) provided measurements with an estimated accuracy of $0.1 \mathrm{~m}$, mainly resulting from uncertainties associated with the visual assessment of blocks gravity centres. In case of breakage of the block, the stopping point considered was the stopping point of the larger resulting fragment, if it could be identified, or the last impact point before breakage, if the block broke in several small pieces. 


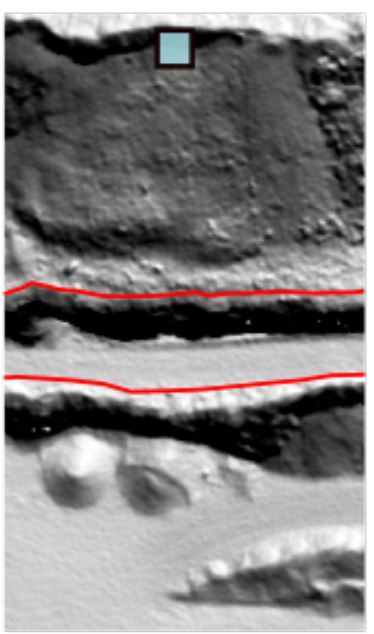

a)

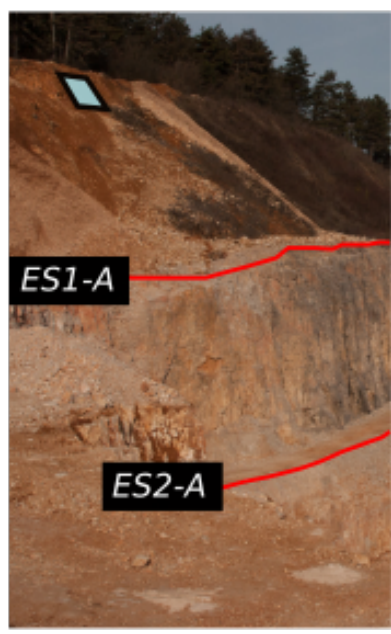

b)

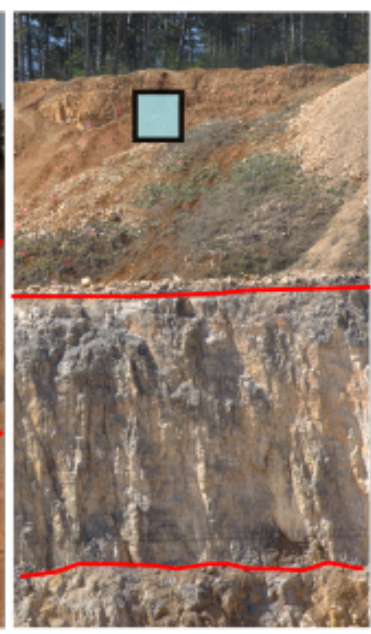

c)

Fig. 5 Views of propagation path A (a: top view, b: side view, c: front view). Blue squares and red lines respectively show the block release zone and evaluation screens location.

Complementary to the stopping points location determination, the trajectory of each block was filmed to provide information on the trajectories and kinematics. Given the configuration of the study site, the safety rules established and the capacities of the cameras, it was not possible to install safely cameras covering the complete propagation paths with sufficient resolution for accurate measurements of the complete trajectories. Consequently, the quantitative analysis of block kinematics focused on measurements at specific locations of the site, called Evaluation Screens (ES) (Figures 5 and 6), using cameras with shooting range focused on the $E S$. The locations of the evaluation screens were chosen in view of measuring the velocities at the transitions between homogeneous zones of the paths in terms of soil and slope properties. These transitions correspond to the location of topographical discontinuities. For Path $A$, the first evaluation screen $(E S 1-A)$ was located at the end of the uphill gentle slope, just before the first rock cut, and the second one (ES2$A$ ) at the top of the downhill rock cut (Figure 5). For Path B, ES1-B is the contour line defined by the elevation of the corridor beginning while ES2-B was located at the downstream extremity of the first sloping track (Figure $6)$. Additionally, two cameras were installed to fully cover the propagation paths. The video footage from these cameras were used for global qualitative analyses of the trajectories since their low resolution did not allow accurate determination of the trajectories.

A protocol for the analysis of the video footage was designed to assess the translation and rotation velocities as well as heights of the blocks when passing through the evaluation screens. 


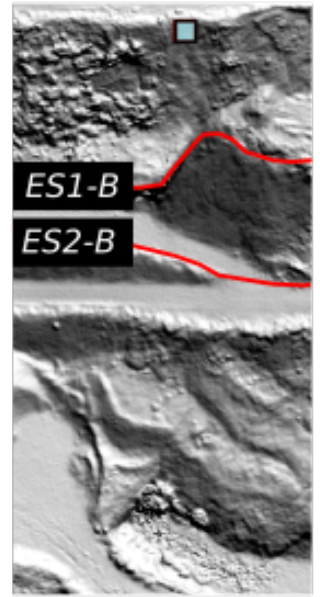

a)

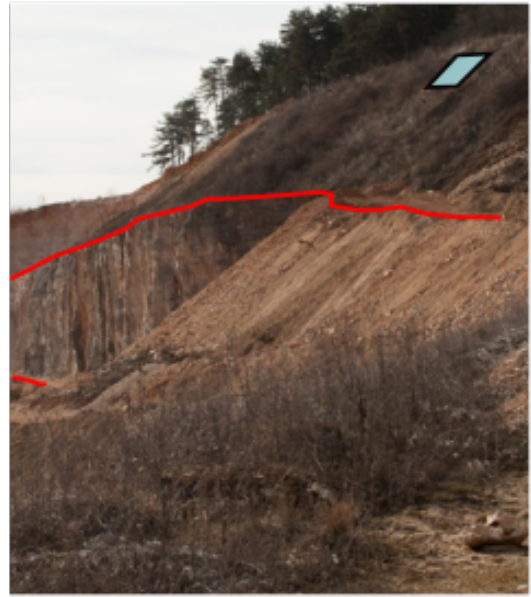

b)

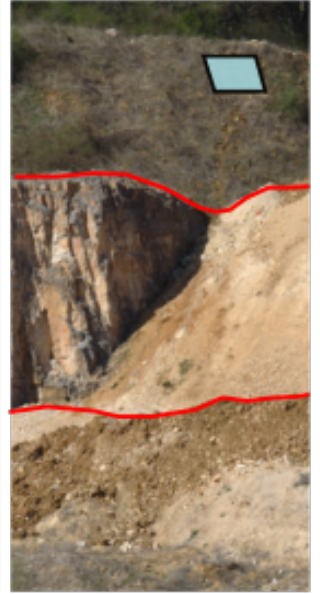

c)

Fig. 6 Views of propagation path B (a: top view, b: side view, c: front view). Blue polygons and red lines respectively show the block release zone and evaluation screens location.

The analysis of the video footage required building a Digital Terrain Model (DTM) of the study site. A DTM (resolution: $0.2 \mathrm{~m}$ ) was thus built from a set of images, taken from a UAV and from the ground. The images were treated using photogrammetry techniques (software Agisoft V1.2.6). 20 control points covering the site, and located in a local coordinate system using a theodolite (Leica TS02), were used in the building process. Two GPS points and one geodesic point were also recorded to georeference the DTM.

The process for assessing the translation velocity consisted, first, in identifying two block positions at the slope surface (just before and just after the evaluation screen). Second, the location of these positions was identified in the three dimensions. Finally, the translation velocity was calculated from this tri-dimensional location and the block travel time between these two positions determined from the digital footage.

This process slightly differed between the case of a block passing through the evaluation screens with significant height, i.e. for a propagation by successive rebounds and flights phases, from the case where the block motion was almost analogous to rolling. In the first case, the block positions just before and just after the evaluation screen correspond to the impact points just before and just after the evaluation screen. In the second one, the two positions were selected to guarantee a distance between them significantly larger than the resolution of the point location detection process, estimated around $0.5 \mathrm{~m}$. The calculation of the tri-dimensional positions from the digital footage was similar for both cases. The images associated with the positions were extracted from the video footage and projected onto the digital terrain model (DTM) so that the tri-dimensional position could be identified. Finally, the calculation of the translation velocity was different. In the case of block motion almost 
analogous to rolling, the velocity at the evaluation screen was assimilated to the mean velocity between the two positions. For significant block passing heights, the exact translation velocity was calculated from the same quantities assuming a parabolic trajectory of the block. Details of this calculation can be found in Bourrier et al. (2012).

Complementary, a qualitative assessment of the passing height was done by visualisation of the video footage and estimation of the passing heights of the block gravity centre, discretized into four classes (contact with soil, small height : approx. $0.5 m$ to $1 m$, medium height : approx. $1 m$ to $3 m$, large height : $>3 m$ approx.). The passing height is defined as the distance along the vertical axis between the soil surface and the block gravity center. The rotational velocity at the evaluation sche block between the two positions with an accuracy of $1 / 8$ revolution.

\section{Experimental results}

The experiments provided data concerning a total of 104 block releases on the two paths (Table 1). All blocks were used to assess the passing probabilities at the evaluation screens. Almost all final positions were also measured, except for two blocks released on Path A due to safety problems. Consequently, the number of measured final positions is smaller than the number of block releases. In addition, several video recordings were missing or not usable for analysis(ES1-A: 14\%, ES2-A: 11\%, ES1-B: 29\%, ES2-B: 33\% ). Indeed, the cameras batteries available had been consumed before the end of the experiments. In addition, the lighting conditions changed during the experiments and, as the experiments were done in limited duration conditions, it had not been possible to stop the experiments to modify the camera settings accordingly.

Block fragmentation occurred more frequently for Path $A$ (16 blocks $33 \%$ of the total) than for Path $B$ (1 block - 1.7\% of the total). For Path $A, 11$ blocks ( $23 \%$ of the total) fragmented into many small parts after a normal impact on a sub-horizontal or horizontal surface (i.e. track or downhill platform). No significantly large fragments propagated after fragmentation, in these cases. A main fragment was identified for the other fragmented blocks. The main fragment did not travel further than approximately $1 m$ for 4 blocks. For one block, a small fragment broke away from the block just after ES1-A and the main fragment propagated after ES2-A. The only block fragmentation observed for Path $B$ is very similar to the latter case. Block fragmentation occurred after $E S 1-B$ and the main fragment propagated after ES2-B. For all fragmented blocks, the initial masses and shapes were considered in the analyses of the influence of these parameters on block propagation. This has no consequence on the observed and discussed trends as only one block per path propagated down the slope after fragmentation. 


\begin{tabular}{ccc} 
& Path A & Path B \\
\hline Number of released blocks & 48 & 56 \\
\hline Number of measured final positions & 46 & 56 \\
\hline Number of trajectories (screen 1) & 38 & 37 \\
\hline Number of trajectories (screen 2) & 17 & 31
\end{tabular}

Table 1 Summary of available data.

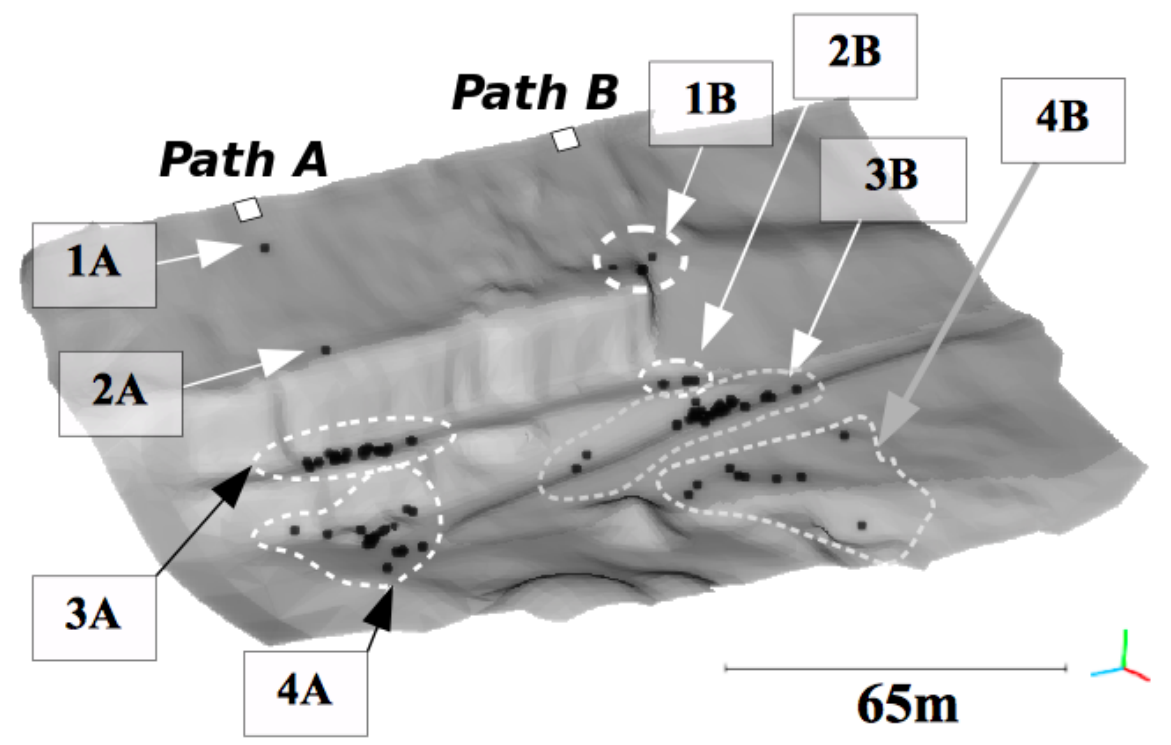

Fig. 7 Final positions of blocks represented using black points. Preferential deposit zones are represented on the right, denoted "A" for Path A and "B" for Path B.

3.1 Spatial distributions of the stopping points

The stopping points shown in Figure 7 suggest differences in terms of propagation distance, preferential deposit zones and lateral dispersion. Visually, it seems that Path $B$ led to longer propagation distances and a larger dispersion. These points are addressed in the following results presentation, before detailing the data collected at the evaluation screens.

For Path A, four deposition zones can be identified (Figure 7). One block propagated few meters after release (zone $1 A$ ) and another one stopped when reaching the flatter part above the first rock cut, after propagating along the uphill slope, (zone 2A). More significant amounts of block stopped on the track, just before ES2-A, (zone $3 A$ ) and on the downhill platform (zone $4 A$ ).

Similarly as for Path A, four deposit zones were identified for Path $B$ (Fig. 7). Five blocks stopped near screen ES1-B (zone $1 B$ ) without reaching the corridor. Three of these blocks remained blocked on the top of the crest of the rock cut, on one side of the corridor. The two others encountered a locally flat surface near the corridor entry, on its other side. Several blocks passed 


\begin{tabular}{ccc} 
Deposit zone & A & B \\
\hline $\mathbf{1}$ & $2.2 \%$ & $8.9 \%$ \\
\hline $\mathbf{2}$ & $2.2 \%$ & $8.9 \%$ \\
\hline $\mathbf{3}$ & $54.3 \%$ & $62.5 \%$ \\
\hline $\mathbf{4}$ & $41.3 \%$ & $19.6 \%$ \\
\hline
\end{tabular}

Table 2 Percentage of deposited blocks in the different zones along paths A and B.

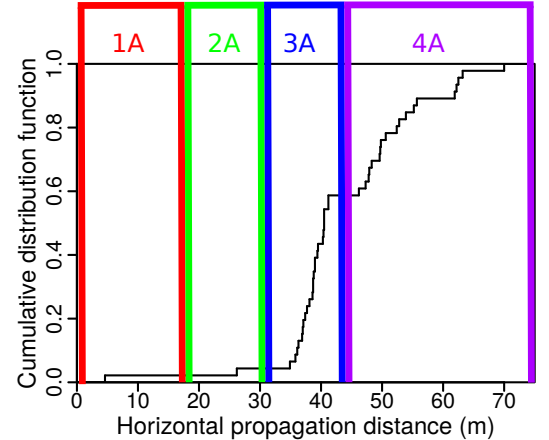

a) Path $A$

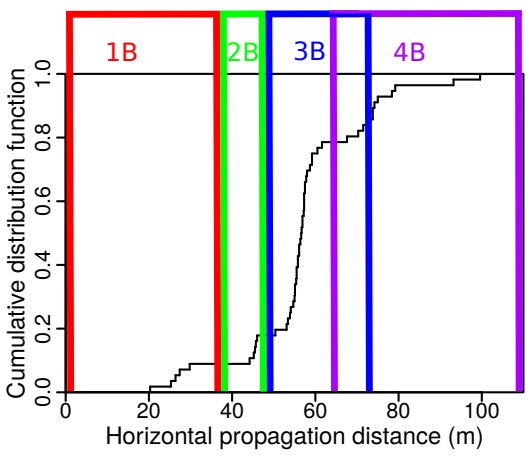

b) Path $B$

Fig. 8 Cumulative distribution functions of block propagation distances for Path $A$ (a) and Path $B(\mathrm{~b})$.

the evaluation screen ES1-B and stopped on the uphill track (zone $2 B$ ) or passed also evaluation screen ES2-B and stopped on the second track (zone $3 B)$. Among these, two were significantly diverted by the gentle slope of the track. Finally, some blocks reached the downhill platform (zone $4 B$ ) .

Table 2 summarizes the percentage of blocks arrested in the different zones.

The deposit zones can also be clearly identified on the cumulative distribution functions (CDFs) of the block propagation distances for Path $A$ and Path $B$ (Figure 8). These CDFs confirm that, for both paths, just few blocks stopped in the first meters of propagation, around $5 \%$ for Path $A$ (zones $1 A$, $2 A$ ) and around $10 \%$ for PathB (zone 1B). A significant amount of blocks reached large propagation distances: around $40 \%$ reached zone $4 A$ and around $20 \%$ reached zone $4 B$. Finally, a majority of blocks stopped at intermediate distances, corresponding to zone $3 A$ for Path $A$ and to zones $2 B$ and $3 B$ for PathB.

As for the lateral dispersion, or deviation, Figure 7 suggested a difference between the two paths. In order to quantify this difference, Figure 9 plots the difference in trajectory orientation, from the release area to the stopping point, with respect to the mean out of the trajectory orientations of all the blocks released on a same path. Two main conclusions raise comparing Path $A$ to Path $B$. First, the distribution is less uniform for Path $B$ with almost 60 $\%$ of the released blocks in the $+/-5^{\circ}$ deviation range. Second, the extreme values are slightly higher for Path $B$. These observations are explained by 


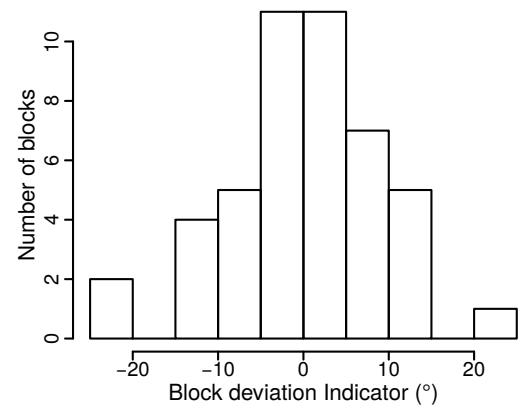

a) Path $A$

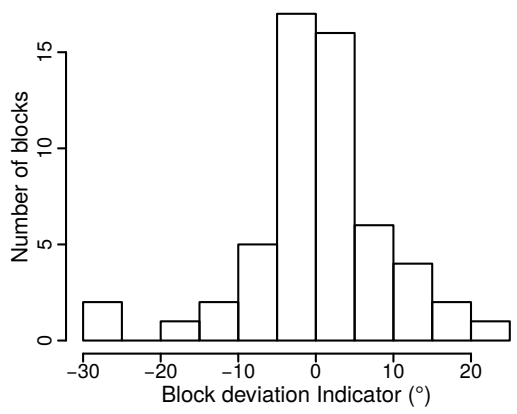

b) Path $B$

Fig. 9 Distribution of block deviation indicators for Path $A$ (a) and Path $B$ (b).

the corridor in Path $B$, which channeling effect globally dominates over the dispersion effect of the two inclined tracks.

\subsection{Trajectories at the evaluation screens}

For Path $A$, the translation velocities of the block at the evaluation screen ES1$A$ vary through a large range $(0.1$ to $8.9 \mathrm{~m} / \mathrm{s}$ - Figure $10 \mathrm{a})$. Their distribution is rather continuous but it is not symmetrical : half of the block velocities are smaller than $2 \mathrm{~m} / \mathrm{s}$ and only $10 \%$ of the velocities are larger than $5 \mathrm{~m} / \mathrm{s}$.

At evaluation screen ES2-A level (Figure 10b), the velocity distribution shows a pronounced bi-modal distribution : $32 \%$ of the velocities are smaller than $5 \mathrm{~m} / \mathrm{s}$ while $68 \%$ are comprised between $18 \mathrm{~m} / \mathrm{s}$ and $19.5 \mathrm{~m} / \mathrm{s}$.

For Path $B$, the translation velocities variation is narrower and the velocity distribution is almost symmetrical at the evaluation screen ES1-B except few block experiencing small velocities, smaller than $2.5 \mathrm{~m} / \mathrm{s}$ for $6 \%$ of the blocks. The velocity distribution exhibits a bi-modal character at evaluation screen ES2-B. Two variation range can be differentiated : $71 \%$ of the velocities range from 0 to $5 \mathrm{~m} / \mathrm{s}$ and $29 \%$ range from $6 \mathrm{~m} / \mathrm{s}$ to $14 \mathrm{~m} / \mathrm{s}$.

Tables 3 provides velocity statistics for both paths and each screen: means, medians, $95 \%$ quantiles and standard deviations. These quantities confirm that the distributions of the velocities are not symmetric: means and medians being clearly different. In addition, the very large standard deviations compared to the means, observed for all evaluation screens, except for ES1-B, confirm the large variability of the velocities.

The comparisons between the velocities at the evaluation screens and the stopping points (Figure 11) do not show significant correlations between these quantities. These comparisons only confirm rather obvious relationships. For example, for Path $A$, only the block passing at ES2-A with large velocities were able to reach large propagation distances (Figure 11c): it corresponds to blocks that bounced near the berm or flew over it. For Path $B$, the velocities 


\begin{tabular}{ccccc} 
& \multicolumn{2}{c}{ Path A } & \multicolumn{2}{c}{ Path B } \\
\hline & ES1-A & ES2-A & ES1-B & ES2-B \\
\hline Passing percentage $(\%)$ & 95.8 & 41.3 & 92.9 & 82.1 \\
\hline Velocity : mean $(m / s)$ & 1.9 & 13.2 & 7.9 & 4.2 \\
\hline Velocity : median $(m / s)$ & 1.1 & 18.6 & 8.1 & 3.1 \\
\hline Velocity : 95\% quantile $(m / s)$ & 6.8 & 19.2 & 11.2 & 8.8 \\
\hline Velocity : standard dev. $(m / s)$ & 8.5 & 8.5 & 2.2 & 3 \\
\hline Coef. of var. $(\%)$ & 447 & 64 & 28 & 71 \\
\hline
\end{tabular}

Table 3 Statistical indicators related with block velocity distributions at the evaluation screens

\begin{tabular}{ccccc} 
& \multicolumn{2}{c}{ Path A } & \multicolumn{2}{c}{ Path B } \\
\hline & ES1-A & ES2-A & ES1-B & ES2-B \\
\hline Passing $(\%)$ & 95.8 soil & 41.3 & 92.9 & 82.1 \\
\hline Mean $(\mathrm{rad} / \mathrm{s})$ & 9.0 & 8.0 & 10.5 & 11.3 \\
\hline Median $(\mathrm{rad} / \mathrm{s})$ & 9.4 & 8.2 & 10.5 & 10.8 \\
\hline Standard dev. $(\mathrm{rad} / \mathrm{s})$ & 2.4 & 2.5 & 3.3 & 3.5 \\
\hline Coef. of var. $(\%)$ & 26 & 31 & 31 & 31 \\
\hline
\end{tabular}

Table 4 Blocks rotational velocities.

of the blocks at the evaluation screen $E S 2-B$ are slightly correlated with those at ES1-B (Figure 11b) because there is no substantial topographical discontinuity between the two evaluation screens. In addition, most of the blocks that reached large propagation distances passed through ES2-A with large velocities (Figure 11c).

The rotational velocities are homogeneously distributed between with values ranging between $3 \mathrm{rad} / \mathrm{s}$ and $20 \mathrm{rad} / \mathrm{s}$. They are globally smaller for Path $A$ than for Path $B$ (Figure 10 - Table 4) and their variability is smaller than the variability of the translation velocities, as shown by the smaller values of coefficients of variations, in particular.

The qualitative analysis of the passing heights highlights an interesting tendency of the blocks to propagate through the evaluation screens at almost nil passing heights (Table 5). For PathA, all the blocks passed through ES1A in contact with the soil and only few of them passed through ES2-A with significant, i.e. medium or large, heights. For PathB, only one block $(2 \%)$ was observed at a significant passing height while all other passed, for both ES1-B and ES2-B, in contact with the soil or at small height. It is interesting to note that, for ES2-A, the very few amount of blocks passing with significant heights seems not consistent with the significant large passing velocities observed (more than $60 \%$ larger than $18 \mathrm{~m} / \mathrm{s}$ ). This apparent inconsistency is due to the fact that many blocks impacted the slope surface just at the location of ES2-A after a long flight phase. For these blocks, we considered the velocity just before impact in the analysis because it was more conservative in terms of protective measures design. 


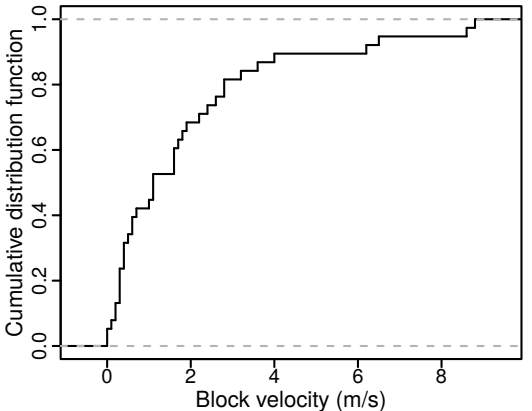

a) ES1-A

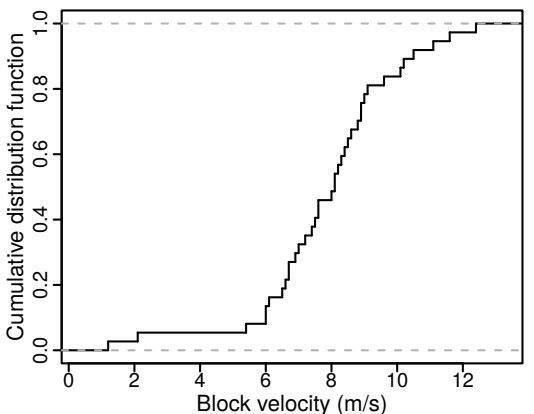

c) ES1-B

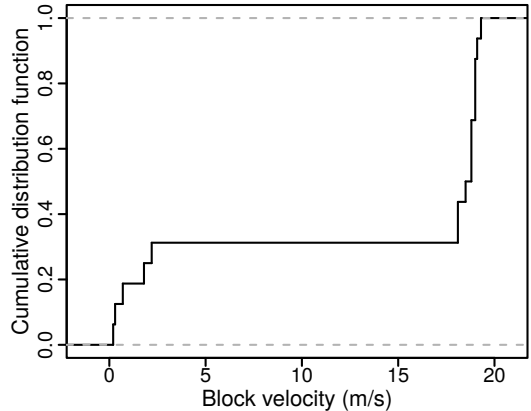

b) ES2-A

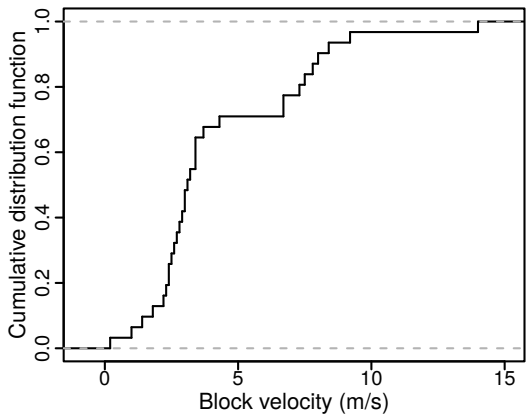

d) ES2-B

Fig. 10 Cumulative distribution functions of block translation velocities (Path A: a,b and Path B: c,d).

Path A

Path B

\begin{tabular}{ccccc}
\hline & ES1-A & ES2-A & ES1-B & ES2-B \\
\hline Contact with soil (\%) & 100 & 65 & 84 & 70 \\
\hline Small passing height : approx. $0.5 m$ to $1 m(\%)$ & 0 & 25 & 14 & 30 \\
\hline Medium passing height : approx. $1 \mathrm{~m}$ to $3 \mathrm{~m}(\%)$ & 0 & 5 & 2 & 0 \\
\hline Large passing heights : approx. $>3 \mathrm{~m}(\%)$ & 0 & 5 & 0 & 0 \\
\hline
\end{tabular}

Table 5 Blocks passing heights.

\subsection{Effect of the blocks characteristics}

The released blocks were characterised in terms of their mass and shape. The influence of both these parameters on the blocks trajectory, classically considered as significant, is addressed in the following.

The map of the blocks stopping points classified according to blocks mass (Figure 13) denotes that most of the blocks presenting higher mass have reached longer propagation distances. On the contrary, some of these heavier boulders stopped earlier compared to small blocks, showing that no univocal relationship exists between the block mass and its propagation distance. How- 


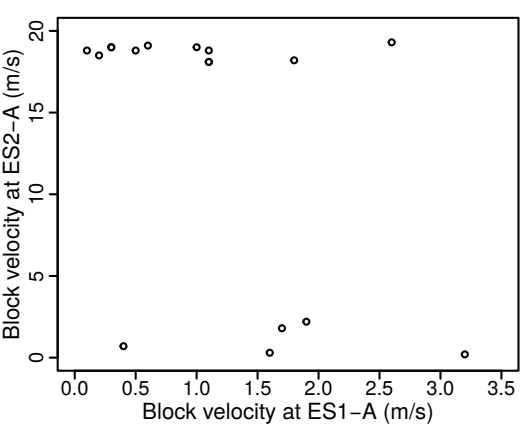

a)

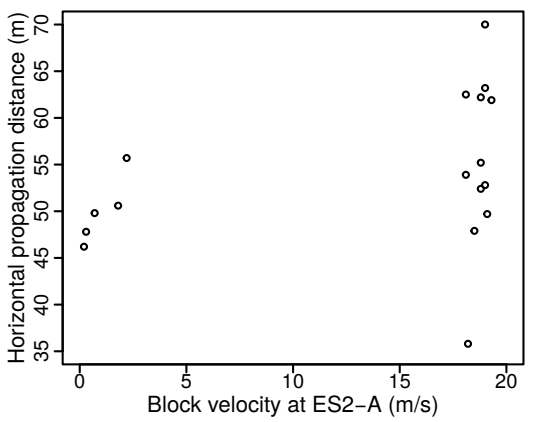

c)

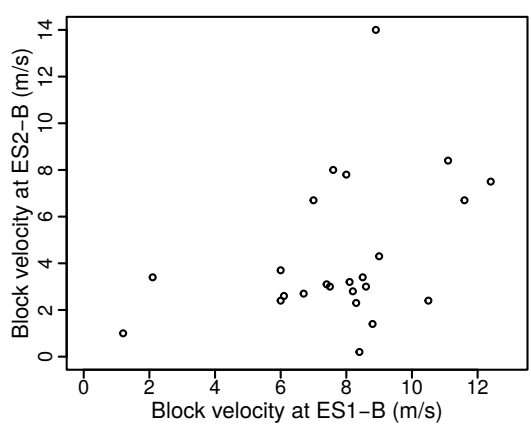

b)

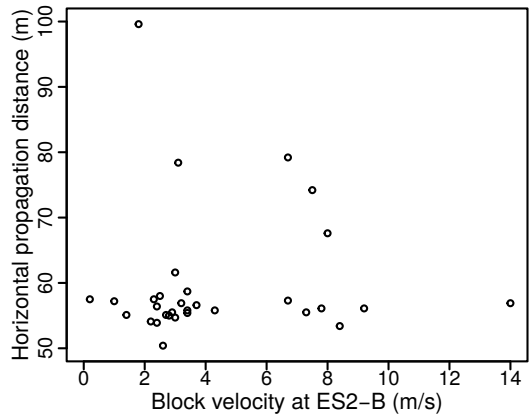

d)

Fig. 11 Relations between block velocities at ES1 and at ES2 and between horizontal propagation distance and block velocity at ES2 (Path A: a, c and Path B: b, d).

ever, these observations remain speculative because the block masses are not evenly distributed (Figure 14).

The analysis of the blocks shape (Fig. 15) highlights that, most of the blocks belong to compact block categories (compact, compact platy, compact bladed and compact elongated) while only few of them are classified as platy, bladed, elongate and very elongate ones. Some shape categories (very platy and very bladed) are not even present. As shape categories proposed are not equiprobable among the blocks used in the experiments, the analysis of the effect of block shape on the propagation remains incomplete. However, the map of the stopping points classified according to the block's shape (Figure 16), shows that the block having more regular shapes - compact blocks in particular but also compact platy and compact bladed blocks - tend to produce longer propagation distances. Indeed, most of the stopping points of these blocks are located in the downhill part of the study site. In comparison, elongate blocks (including compact elongate, elongate and very elongate) seem to reach smaller propagation distances than compact ones. Concerning other block categories, no conclusion should be drawn due to the small number of concerned blocks. 


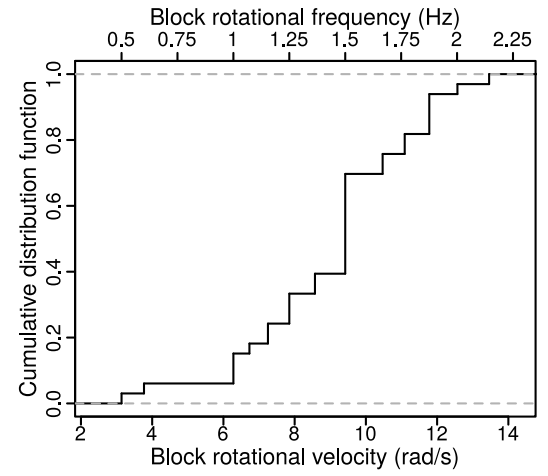

a) ES1-A

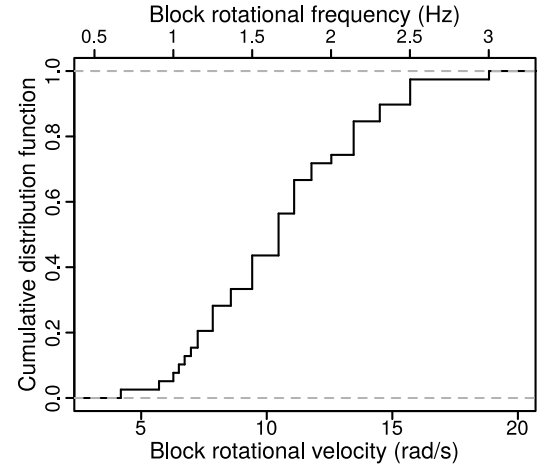

c) ES1-B

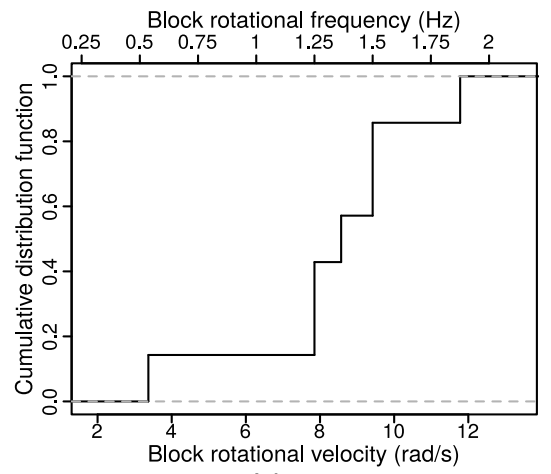

b) ES2-A

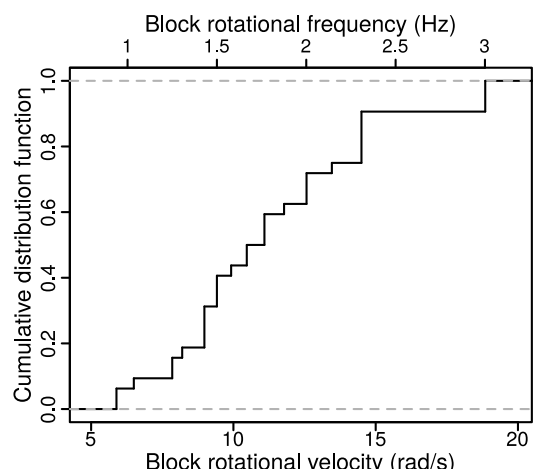

d) ES2-B

Fig. 12 Cumulative distribution functions of the blocks rotational velocity (Path A: a,b and Path B: c,d).

\section{Discussion}

\subsection{Prevailing effects}

The analysis of the effects prevailing over the block propagation was done with the objective to identify the leading parameters as well as the physical processes which should be considered for accurately modeling the blocks propagation in this study site.

The spatial distribution of the stopping points emphasizes clear preferential deposit zones of the blocks at the topographical discontinuities and delimits clear preferential propagation paths mainly driven by the global characteristics of the propagation path. In particular, topography determines simple but essential characteristics of the blocks propagation such as the zones where the block cannot propagate, e.g. zones separated from the main corridor by impassable obstacles. Topography also determines the zones where blocks will preferentially stop, for instance on nearly horizontal surfaces at the toe of rock cuts provided that soil properties guarantee a minimum block energy dissipa- 

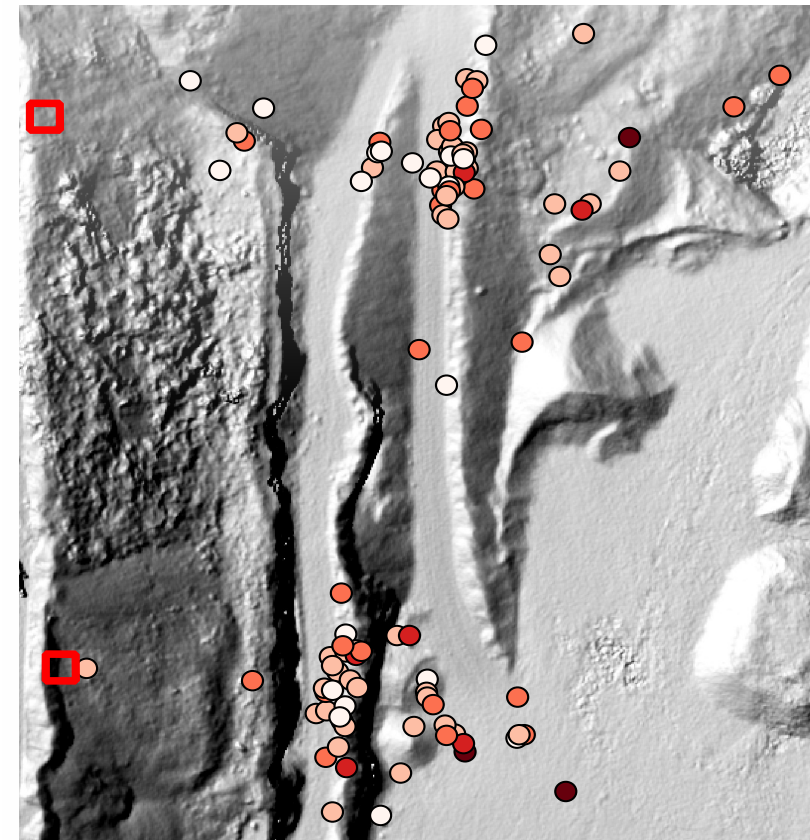

\begin{tabular}{|c|}
\hline Release zone \\
\hline Block mass (kg) \\
\hline O $150-500$ \\
\hline $500-850$ \\
\hline $850-1200$ \\
\hline $1200-1550$ \\
\hline $1550-1900$ \\
\hline
\end{tabular}

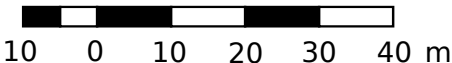

Fig. 13 Map of the blocks stopping points classified according to the mass of the blocks.

tion at impact, as well as against obstacles such as the berms. Consequently, these experimental results confirm that topography is a parameter of major influence on block propagation and stopping.

Nevertheless, topography is not the only parameter with influence on block propagation, as illustrated by the substantial variability in the block trajectories for both propagation paths. This is revealed by the distribution of both the stopping points and the velocities at the evaluation screens. In addition, the experiments showed that there exist several possibilities for the blocks to get through a local topographical discontinuity. For example, at the level of Evaluation screen 2 for Path $B$, the blocks can bounce on the middle of the track and jump over the berm or make several small bounces on the track to roll over the berm. This variability shows that many other factors than topography influence block propagation. 


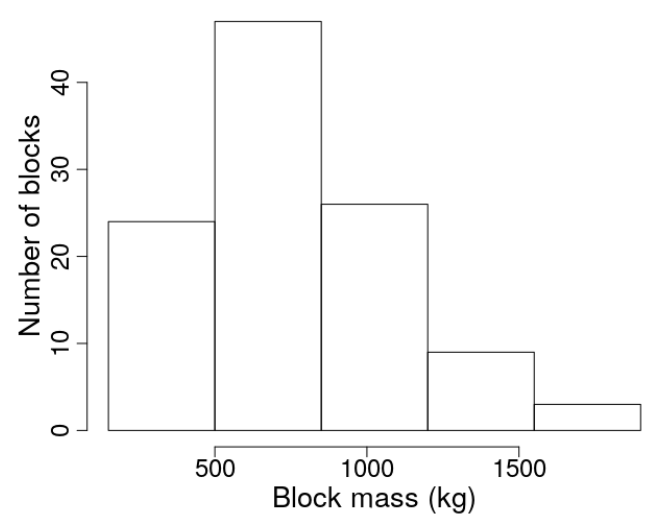

Fig. 14 Distribution of the mass of the blocks according to the classes used to analyse the influence of the mass of the blocks on the stopping points.

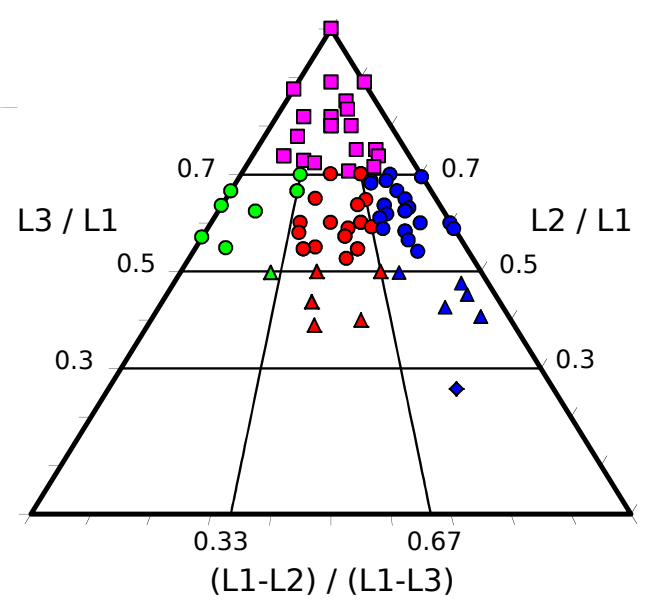

Shape (Sneed \& Folk, 1958)
$\square$ Compact
$\triangle$ Platy
A Bladed
A Elongate
- Compact Platy
- Compact Bladed
- Compact Elongate
- Very Elongate

Fig. 15 Shapes of the blocks used in the experiments.

Among the various parameters, the soil properties on both paths are rather homogeneous for each type of soil (quarry waste, rock cut, track,...). The variability in the trajectories cannot be explained by the variability in the soil properties inside each homogeneous zone. As for the blocks characteristics, the analysis of the experimental results suggested a qualitative influence of the block shape on the propagation distance but did not reveal any trend in relation to the block mass. Despite the speculative character of the latter observation, it is worth noting that it contrasts with the commonly believed influence of the mass on the propagation distance of the blocks. As regards to the influence of block shape, it is also interesting to mention that a reduced 

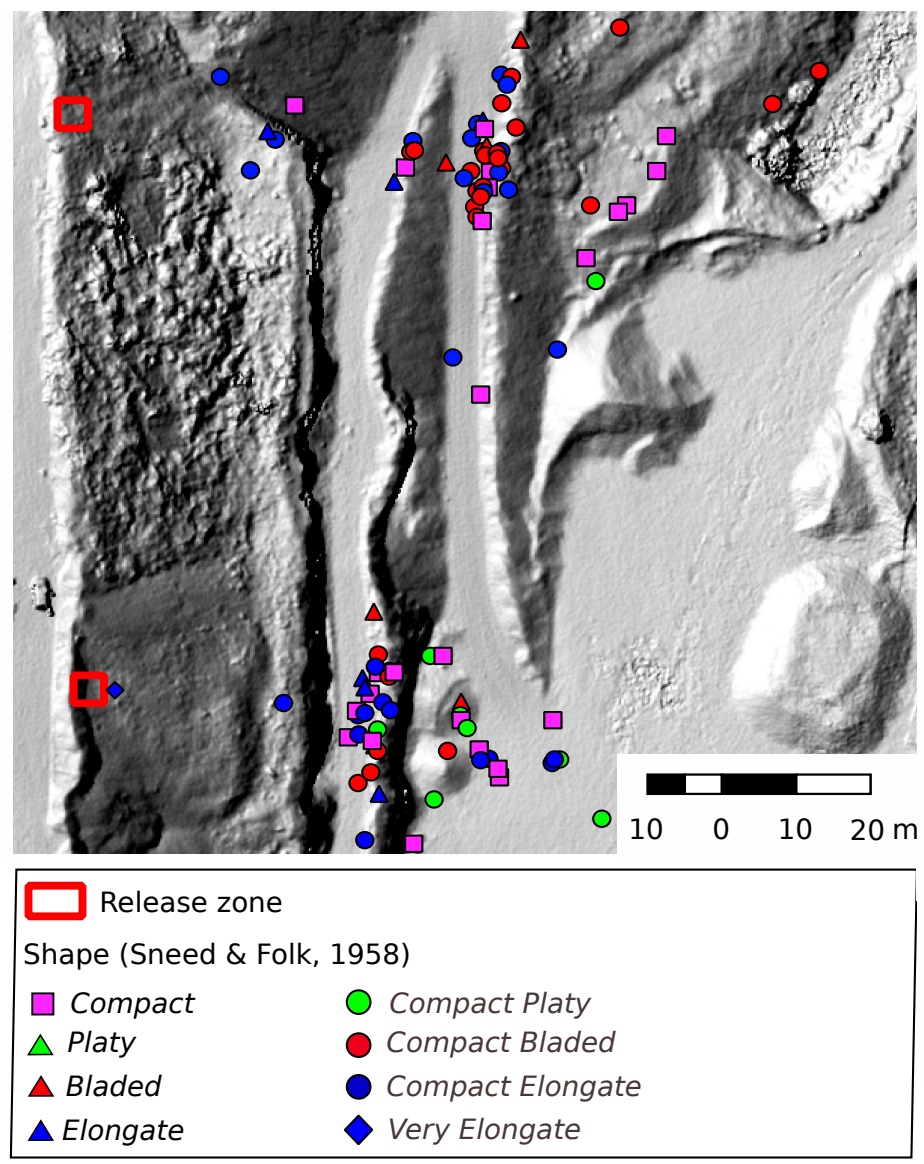

Fig. 16 Map of the blocks stopping points classified according to the shape categories.

influence of block shape has already been observed in a similar context (Preh et al. 2015).

In addition to these rather well characterised parameters, the block propagation may also depend on other parameters. The variability may thus stem from other parameters that were not measured in these experiments because it is not practically feasible at the site scale. These parameters concern local features, i.e. in the impact point vicinity and small scale quantities such as, in particular, the details of block shape, the orientation of the block at impact, the local topography or locally specific properties of the soil.

More generally, the variability in the trajectories is also thought to result from an interdependence between the various parameters in terms of their influence on the block propagation. In this study, three main parameters were considered: topography, block mass and block shape. It is believed that the influence of one parameter depends on the value of the two others. There is a competition between the parameters in their ability in influencing the block 
propagation at the local scale. For instance, a high topographical discontinuity (berm for example), may modify or neutralise the influence of the block mass or shape on the block propagation. This results in a variability in the trajectories on this site and suggests that studies focusing on the influence of one parameter on sites where the other parameters are uniform provide qualitative evidences and quantitative trends that should not be extrapolated for cases in other situations.

In summary, in the context of these controlled experiments, the propagation process is driven by topography and several local parameters. The latter parameters, which entail significant variability of the results, cannot be easily identified at the slope scale for propagation modelling purposes in operational contexts. They can be identified but their relative influences can hardly be quantified.

\subsection{Towards block propagation modelling}

The analysis of the processes leading block propagation on the study site provides important information to model this propagation and to assess the relevance of block propagation models in this site. In particular, the experiments showed that block propagation is mainly influenced by topography and that substantial variability of the block trajectories exists.

First, as topography plays a major role on block propagation, it is mandatory to perform propagation simulations with 3D Digital Terrain Models, or $2 \mathrm{D}$ profiles if $3 \mathrm{D}$ simulations are not feasible for technical or economical reasons, that represent topography in details. In particular, the quality of the representation of the topographical discontinuities is essential because these continuities induce propagation options. Automatically generated digital terrain models (DTM) are not necessarily relevant in that sense, even for high resolution DTM. They have to be checked and improved manually by expert knowledge. For example, the presence of slope discontinuities (ledges, mounds, etc.) has to be checked and the terrain model has to be modified if the slope discontinuities are not accurately represented. Such problem can occur if the resolution of the DTM is too low or if the DTM format is not adapted (for example, raster DTM may not allow reproducing sharp slope changes (Lambert et al. 2013).

Second, even if the influence of soil properties, block mass and block shape on the propagation has not been clearly identified in the experiments, these parameters are classically identified in the literature for playing an important role on block propagation (Bourrier and Hungr 2013). They thus have to be quantified in the field and integrated into the modelling process. This stage is classical in block propagation modelling but it remains important. A field survey of the soil mechanical properties is thus mandatory to identify homogeneous zones and quantify the model parameters associated with soil properties at the global scale of the study site. Quantitative procedures to assess block mass and shape have also to be performed. 
In addition to the above mentioned procedures, it is of primary importance to traduce the variability of the trajectories. As the soil properties are rather homogeneous for each type of soil (quarry waste, rock cut, track,...), the variability cannot be fully attributed to this parameter. In addition, the analyses showed that block mass or shape were not the main causes of variability. On the contrary, the experiments showed the substantial influence of local conditions in the vicinity of the impact point, that cannot be easily quantified in the field, on the propagation. These conditions are of several nature. They may be related with the local terrain properties, both in terms of geometry and mechanical properties, and with the local impact conditions (block orientation, in particular). Random variables sampled from calibrated distributions (Li and Lan 2015) are classicaly used to account for the variability induced by the latter conditions. The propagation models either use one single random variable that traduces the complete variability associated with all local parameters or different random variables associated with the different local sources of variability. The main difficulty related with the practical use of such probabilistic modelling approaches is the calibration of the distributions of the random variables chosen.

Finally, the conclusions drawn from the experiments illustrate the misleading character of several classical practices for the qualitative assessment of the relevance and accuracy of propagation simulations. For example, as there is no clear relationships between the block size, shape and the propagation distance, it is not possible to assess the quality of simulations from the presence of size or shape sorting in the deposit. In the same vein, the local parameters influenced the block trajectories in such an important way that no clear relationships exist between the velocities at the different evaluation screens and the stopping points (Figure 11). The consequence is that, contrary to our initial expectations, it is not possible to identify archetype trajectories at the slope scale. As no archetype trajectories can be identified at the slope scale, the presence of such archetypes in the simulations does not guarantee their relevance. Consequently, the assessment of the relevance and accuracy of propagation simulations remains limited to the comparison between the simulations and previous rockfall events on the site or on similar study sites. This assessment can consist of comparing the preferential deposit zones, or the preferential transit zones. For that purpose, the mapping of the deposited blocks in the sites or the use of dendrogeomorphology techniques to identify preferential transit zone constitute valuable tools.

\section{Conclusion}

The dataset obtained from the analysis of block propagation on the two propagation paths allowed to assess the complexity stemming from the presence of topography discontinuity and of combinations of gentle slopes and soft soils, favoring block motion almost analogous to rolling, in the study site. This 
dataset could be used by researchers or practitioners that would like to assess the relevance of propagation modelling tools on such a complex study site.

On both propagation paths, preferential transit and deposit zones were identified. Complementary, significant variability of the stopping points and of the block kinematics at the evaluation screens was observed. This variability revealed not to be clearly correlated with specific block properties, except a qualitative influence of block shape on the propagation distance. In addition, the joint analysis of the block kinematics evolution between the evaluation screens and of the stopping points did not allow to identify archetype trajectories at the slope scale.

While the presence of preferential transit and deposit zones highlights the substantial influence of topography, the absence of archetype trajectories at the slope scale illustrates the effects of local conditions, e.g. block shape, orientation, local topography, specificity of soil properties, in the vicinity of the impact point. For example, in the presence of a topographical discontinuity different propagation options exist but the occurrence of one of these options depends mainly on local conditions.

Given the predominant effect of topography, in particular of topographical discontinuities, block propagation simulations should be based on accurate digital terrain models that traduces these discontinuities. In addition, the predominant effect of local conditions, whose deterministic quantification would require substantial field effort, tends to favor the use of probabilistic modelling of block propagation using sound random variables that traduce the influence of local conditions. Finally, the absence of archetype trajectories and of correlations between block properties and propagation entails difficulties in evaluating the quality of block propagation simulations.

The detailed analysis of the propagation on the study site and of the associated prevailing effects provides additional information of primary importance for the researchers or practitioners that would like to assess the relevance of propagation modelling tools on such a complex study site. It also highlights two major challenges in the field of block propagation modelling: the identification of sound random variables for the probabilistic modelling of block propagation and the assessment of propagation simulations results relevance based on statistical comparisons with relevant field surveys or experiments.

Acknowledgements The topography of the study site and the results of the experiments are available in supplementary materials attached to this article or upon request (franck.bourrier@inrae.fr).

This research work has been held in the context of the french national project C2ROP, which provided financial support for the experiments. The authors would like to thank all the partners involved in these experiments, including research laboratories and institutes, stakeholders, and private engineering companies.

\section{Conflict of interest}

The authors declare that they have no conflict of interest. 


\section{References}

Abellan A, Calvet J, Vilaplana JM, Blanchard J (2010) Detection and spatial prediction of rockfalls by means of terrestrial laser scanner monitoring. Geomorphology 119(3):162 - 171, DOI https://doi.org/10.1016/j.geomorph.2010.03.016, URL http://www.sciencedirect.com/science/article/pii/S0169555X10001224

Agliardi F, Crosta GB, Frattini P (2009) Integrating rockfall risk assessment and countermeasure design by $3 \mathrm{~d}$ modelling techniques. Natural Hazards and Earth System Sciences 9(4):1059-1073, DOI 10.5194/nhess-9-1059-2009, URL https://www.nat-hazards-earthsyst-sci.net/9/1059/2009/

Asteriou P, Tsiambaos G (2018) Effect of impact velocity, block mass and hardness on the coefficients of restitution for rockfall analysis. International Journal of Rock Mechanics and Mining Sciences 106:41 - 50, DOI https://doi.org/10.1016/j.ijrmms.2018.04.001, URL http://www.sciencedirect.com/science/article/pii/S1365160917309917

Blott SJ, Pye K (2008) Particle shape: a review and new methods of characterization and classification. Sedimentology 55(1):31-63, DOI 10.1111/j.1365-3091.2007.00892.x, URL https://onlinelibrary.wiley.com/doi/abs/10.1111/j.1365-3091.2007.00892.x, https://onlinelibrary.wiley.com/doi/pdf/10.1111/j.1365-3091.2007.00892.x

Bonneau DA, Hutchinson DJ, DiFrancesco PM, Coombs M, Sala Z (2019) Threedimensional rockfall shape back analysis: methods and implications. Natural Hazards and Earth System Sciences 19(12):2745-2765, DOI 10.5194/nhess-19-2745-2019, URL https://www.nat-hazards-earth-syst-sci.net/19/2745/2019/

Bourrier F, Hungr O (2013) Rockfall Engineering, ISTE-Wiley, chap Rockfall Dynamics: A Critical Review of Collision and Rebound Models, pp 175-209. DOI 10.1002/9781118601532.ch6

Bourrier F, Dorren L, Nicot F, Berger F, Darve F (2009) Toward objective rockfall trajectory simulation using a stochastic impact model. Geomorphology 110(3):68 - 79, DOI https://doi.org/10.1016/j.geomorph.2009.03.017, URL http://www.sciencedirect.com/science/article/pii/S0169555X09001251

Bourrier F, Berger F, Tardif P, Dorren L, Hungr O (2012) Rockfall rebound: comparison of detailed field experiments and alternative modelling approaches. Earth Surface Processes and Landforms 37(6):656-665, DOI 10.1002/esp.3202, URL https://onlinelibrary.wiley.com/doi/abs/10.1002/esp.3202, https://onlinelibrary.wiley.com/doi/pdf/10.1002/esp.3202

Caviezel A, Demmel SE, Ringenbach A, Bühler Y, Lu G, Christen M, Dinneen CE, Eberhard LA, von Rickenbach D, Bartelt P (2019) Reconstruction of four-dimensional rockfall trajectories using remote sensing and rock-based accelerometers and gyroscopes. Earth Surface Dynamics 7(1):199-210, DOI 10.5194/esurf-7-199-2019, URL https://www.earthsurf-dynam.net/7/199/2019/

Chau K, Wong R, Lee C (1998) Rockfall problems in hong kong and some new experimental results for coefficients of restitution. International Journal of Rock Mechanics and Mining Sciences 35(4):662 - 663, DOI https://doi.org/10.1016/S0148-9062(98)00023-0, URL http://www.sciencedirect.com/science/article/pii/S0148906298000230

Corominas J, Copons R, Moya J, Vilaplana JM, Altimir J, Amigó J (2005) Quantitative assessment of the residual risk in a rockfall protected area. Landslides 2(4):343-357, DOI 10.1007/s10346-005-0022-z, URL https://doi.org/10.1007/s10346-005-0022-z

Dewez TJ, Rohmer J, Regard V, Cnudde C (2013) Probabilistic coastal cliff collapse hazard from repeated terrestrial laser surveys: case study from Mesnil Val (Normandy, northern France). Journal of Coastal Research 65(sp1):702 - 707, DOI 10.2112/SI65-119.1, URL https://doi.org/10.2112/SI65-119.1

Dincer I, Orhan A, Frattini P, Crosta GB (2016) Rockfall at the heritage site of the tatlarin underground city (cappadocia, turkey). Natural Hazards 82(2):1075-1098, DOI 10.1007/s11069-016-2234-z, URL https://doi.org/10.1007/s11069-016-2234-z

Dorren LKA, Berger F, Putters US (2006) Real-size experiments and 3-d simulation of rockfall on forested and non-forested slopes. Natural Hazards and Earth System Sciences 6(1):145-153, DOI 10.5194/nhess-6-145-2006, URL https://www.nat-hazardsearth-syst-sci.net/6/145/2006/ 
Dussauge-Peisser C, Helmstetter A, Grasso JR, Hantz D, Desvarreux P, Jeannin M, Giraud A (2002) Probabilistic approach to rock fall hazard assessment: potential of historical data analysis. Natural Hazards and Earth System Sciences 2(1/2):15-26, DOI 10.5194/nhess-2-15-2002, URL https://www.nat-hazards-earth-syst-sci.net/2/15/2002/

Garcia B, Villard P, Richefeu V, Daudon D (2017) Experimental and dem analysis of the dissipation involved in the collision of a boulder with a substratum. EPJ Web Conf 140:16010, DOI 10.1051/epjconf/201714016010, URL https://doi.org/10.1051/epjconf/201714016010

Giani GP, Giacomini A, Migliazza M, Segalini A (2004) Experimental and theoretical studies to improve rock fall analysis and protection work design. Rock Mechanics and Rock Engineering 37(5):369-389, DOI 10.1007/s00603-004-0027-2, URL https://doi.org/10.1007/s00603-004-0027-2

Glover J (2015) Rock shape and its role in rockfall dynamics. PhD thesis, DurhamUniversity

Guzzetti F, Reichenbach P, Wieczorek GF (2003) Rockfall hazard and risk assessment in the Yosemite Valley, California, USA. Natural Hazards and Earth System Science 3(6):491503, URL https://hal.archives-ouvertes.fr/hal-00299059

Hantz D (2011) Quantitative assessment of diffuse rock fall hazard along a cliff foot. Natural Hazards and Earth System Sciences 11(5):1303-1309, DOI 10.5194/nhess-11-1303-2011, URL https://www.nat-hazards-earth-syst-sci.net/11/1303/2011/

Heim A (1932) Der Bergsturz und Menschenleben. Fretz und Wasmuth Verlag p 218

Hu J, Li S, Li L, Shi S, Zhou Z, Liu H, He P (2017) Field, experimental, and numerical investigation of a rockfall above a tunnel portal in southwestern china. Bulletin of Engineering Geology and the Environment DOI 10.1007/s10064-017-1152-y

Jaboyedoff M, Labiouse V (2011) Technical note: Preliminary estimation of rockfall runout zones. Natural Hazards and Earth System Sciences 11(3):819-828, DOI 10.5194/nhess11-819-2011, URL https://www.nat-hazards-earth-syst-sci.net/11/819/2011/

Labiouse V, Heidenreich B (2009) Half-scale experimental study of rockfall impacts on sandy slopes. Natural Hazards and Earth System Sciences 9(6):1981-1993, DOI 10.5194/nhess9-1981-2009, URL https://www.nat-hazards-earth-syst-sci.net/9/1981/2009/

Lambert S, Bourrier F, Toe D (2013) Improving three-dimensional rockfall trajectory simulation codes for assessing the efficiency of protective embankments. International Journal of Rock Mechanics and Mining Sciences 60:26 - 36, DOI https://doi.org/10.1016/j.ijrmms.2012.12.029, URL http://www.sciencedirect.com/science/article/pii/S1365160912002572

Leine RI, Schweizer A, Christen M, Glover J, Bartelt P, Gerber W (2014) Simulation of rockfall trajectories with consideration of rock shape. Multibody System Dynamics 32(2):241-271, DOI 10.1007/s11044-013-9393-4, URL https://doi.org/10.1007/s11044013-9393-4

Li L, Lan H (2015) Probabilistic modeling of rockfall trajectories: a review. Bulletin of Engineering Geology and the Environment 74(4):1163-1176, DOI 10.1007/s10064-0150718-9, URL https://doi.org/10.1007/s10064-015-0718-9

Lu G, Caviezel A, Christen M, Demmel SE, Ringenbach A, Bühler Y, Dinneen CE, Gerber W, Bartelt P (2019) Modelling rockfall impact with scarring in compactable soils. Landslides DOI 10.1007/s10346-019-01238-z, URL https://doi.org/10.1007/s10346-01901238-z

Macciotta R, Martin CD (2019) Preliminary approach for prioritizing resource allocation for rock fall hazard investigations based on susceptibility mapping and efficient three-dimensional trajectory modelling. Bulletin of Engineering Geology and the Environment 78(4):2803-2815, DOI 10.1007/s10064-018-1279-5, URL https://doi.org/10.1007/s10064-018-1279-5

Preh A, Mitchell A, Hungr O, Kolenprat B (2015) Stochastic analysis of rock fall dynamics on quarry slopes. International Journal of Rock Mechanics and Mining Sciences 80:57 66, DOI https://doi.org/10.1016/j.ijrmms.2015.09.010

Rabatel A, Deline P, Jaillet S, Ravanel L (2008) Rock falls in high-alpine rock walls quantified by terrestrial lidar measurements: A case study in the mont blanc area. Geophysical Research Letters 35(10), DOI 10.1029/2008GL033424, URL https://agupubs.onlinelibrary.wiley.com/doi/abs/10.1029/2008GL033424, https://agupubs.onlinelibrary.wiley.com/doi/pdf/10.1029/2008GL033424 
Sarro R, Riquelme A, Lopez-Davalillo JC, Mateos R, Toms R, Pastor Navarro J, Cano M, Herrera G (2018) Rockfall simulation based on uav photogrammetry data obtained during an emergency declaration: Application at a cultural heritage site. Remote Sensing 10:1923, DOI 10.3390/rs10121923

Sneed ED, Folk RL (1958) Pebbles in the lower colorado river, texas a study in particle morphogenesis. The Journal of Geology 66(2):114-150, DOI 10.1086/626490, URL https://doi.org/10.1086/626490, https://doi.org/10.1086/626490

Spadari M, Giacomini A, Buzzi O, Fityus S, Giani G (2012) In situ rockfall testing in new south wales, australia. International Journal of Rock Mechanics and Mining Sciences 49:84 - 93, DOI https://doi.org/10.1016/j.ijrmms.2011.11.013, URL http://www.sciencedirect.com/science/article/pii/S1365160911001997

van Veen M, Hutchinson D, Kromer R, Lato M, Edwards T (2017) Effects of sampling interval on the frequency - magnitude relationship of rockfalls detected from terrestrial laser scanning using semi-automated methods. Landslides 14(5):1579-1592, DOI 10.1007/s10346-017-0801-3, cited By 18 